\title{
GROUPOÏDE FONDAMENTAL ET D'HOLONOMIE DE CERTAINS FEUILLETAGES RÉGULIERS
}

\author{
M. C. LASSO DE LA VEGA
}

\begin{abstract}
Let $M$ be a manifold with a regular foliation $\mathcal{F}$. We recall the construction of the fundamental groupoid and the homotopy groupoid associated to $\mathcal{F}$. We describe some interesting particular cases and give some gluing techniques. We characterize the cases where these groupoids are Hausdorff spaces.

We study in particular both groupoids associated to foliations with Reeb components.
\end{abstract}

\section{Introduction}

Dans le premier paragraphe on rappelle la construction du groupoide fondamental, $\Pi_{1}(\mathcal{F})$, et celle du groupoïde d'holonomie, $H(\mathcal{F})$, associés à un feuilletage régulier, qui ont déjà été faites par J. Phillips [Phi] et Hi. E. Winkelnkemper [Win] respectivernent. On étudie quelques cas particuliers intéressants et on explicite quelques techniques de recollement. On donne des conditions nécessaires et suffisantes pour que $\Pi_{1}(\mathcal{F})$ et $H(\mathcal{F})$ soient separés.

Aux deuxième et troisième paragraphes on construit en détail le groupoïde fondamental et d'homotopie des feuilletages qui contiennent des composantes de Reeb.

Je voudrais remercier le Professeur Gilbert Hector pour m'avoir proposé ce travail ainsi que pour le temps, la patience et l'aide qut'il m'a consacrés.

\section{Le groupoïde fondamental et le groupoïde d'holonomie}

1.1. Construction du groupoïde fondamental $\Pi_{1}(\mathcal{F})$.

Soit $\mathcal{F}$ un feuilletage régulier de dimension $p$ sur une varieté $M$ de dimension $p+n$.

Considérons le diagramme commutatif

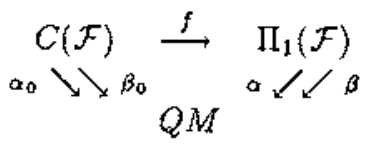


défini par les données suivantes:

(a) $C(\mathcal{F})$ étant l'espace des chemins intégraux de $\mathcal{F}$ muni de la topologie compacte-ouverte, les projections source $\alpha_{0}$ et but $\beta_{0}$ sont continues et ouvertes;

(b) $\Pi_{1}(\mathcal{F})$ est le quotient de $C(\mathcal{F})$ par la relation d'équivalence ouverte qui identifie les chemins à extrémités fixes qui sont homotopes sur la feuille qui les contient; $f$ est la projection quotient naturelle et les applications $\alpha$ et $\beta$ obtenues par passage au quotient sont également continues et ouvertes;

(c) la composition, (resp. l'inversion), usuelle des chemins définit une loi de composition continue $m$, (resp. un homéomorphisme $i$ ), de $\Pi_{1}(\mathcal{F})$ qui font de $\Pi_{1}(\mathcal{F})$ un groupoïde localement compact, dont l'espace des unités $\Gamma_{0}$ est égal à $M$ (identifié à l'ensemble de classes de chemins constants);

(d) la topologie sur $C(\mathcal{F})$ définit sur $\Pi_{1}(\mathcal{F})$ une structure de variété différentiable de dimension $2 p+n$ [Phi], pour laquelle $\alpha$ et $\beta$ sont des submersions, $m$ est différentiable et $i$ est un difféomorphisme. Bref $\Pi_{1}(\mathcal{F})$ devient, un groupoïde de Lie.

On notera Iso $\Pi_{1}(\mathcal{F})$ l'ensemble de classes de lacets de $\Pi_{1}(\mathcal{F})$. C'est un sous-groupoïde invariant qu'on appellera groupoïde d'isotropie de $\Pi_{1}(\mathcal{F})$.

Remarque. En particulier si $n=0$, c'est-à-dire si $\mathcal{F}$ est le feuilietage trivial ayant une seule feuille égale à $M$, la construction précédente se réduit à celle du groupoïde fondamental de $M$, noté $\Pi_{1}(M) \rightrightarrows M$, pour lequel $\alpha$ et $\beta$ sont des fibrations localement triviales transverses l'une à l'autre et ayant pour fibre le revêtement universel de $M$.

Dans le cas génèral on retrouve cette situation au-dessus de chaque feuille $F \in \mathcal{F}$, c'est-à-dire on a un diagramme commutatif:

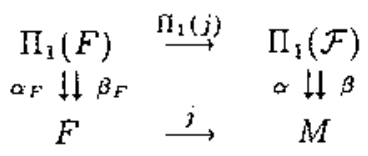

où l'application $\Pi_{1}(j)$, induite par $j$, est une inmersion dont l'image est égale à $\alpha^{-1}(F)=\beta^{-1}(F)$.

\subsection{Groupoïde fondamental et groupoïde d'holonomie.}

Le groupoide d'holonomie $H(\mathcal{F})$ de $\mathcal{F}$ peut être construit de façon tout à fait analogue à celle de $\Pi_{1}(\mathcal{F})$, la seule différence étant que la relation d'équivalence utilisée est strictement plus fine: deux chemins $\gamma_{1}$ et $\gamma_{2}$ sur le mème feuille et de mêmes extrémités, sont équivalents si le germe d'holonomie du lacet $\gamma_{2}^{-1} * \gamma_{1}$ est trivial. On a done une factorisation:

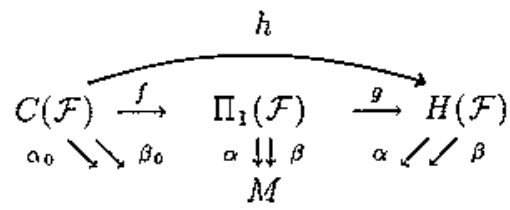


et $g$ est un morphisme surjectif de groupoïdes de Lie, qui est en fait un difféornorphisme local, et que pour chaque $u \in M$ induit un revêtement des $\alpha$-fibres (resp. $\beta$-fibres) respectives.

\subsection{Cas particuliers.}

(a) Action d'un groupe de Lie.

Une action $\phi: G \times M \longrightarrow M$ d'un groupe de lie $G$, connexe, sur une varieté $M$ définit un groupoïde de Lie $\Gamma_{\phi}$ en posant:

$$
\begin{gathered}
\alpha(g, u)=u \quad \beta(g, u)=\phi(g, u)=g(u) ; \\
m\left[\left(g_{2}, u_{2}\right),\left(g_{1}, u_{1}\right)\right]=\left(g_{1} \cdot g_{2}, u\right) \text { si } u_{2}=\phi\left(g_{1}, u_{1}\right) ; \\
i(g, u)=\left(g^{-3}, g u\right) .
\end{gathered}
$$

Les orbites de ce groupoïde, c'est-⿳亠㐅े-dire, les ensembles $\beta\left(\alpha^{-1}(u)\right)=$ $\alpha\left(\beta^{-1}(u)\right)$, coincident avec les orbites de l'action $\phi$ et définissent sur $M$ un feuilletage $\mathcal{F}_{\phi}$, en général singulier (ou de Stefan).

Si l'action est localement libre, (donc $\mathcal{F}_{\phi}$ est régulier), en remplaçant $G$ par son revêtement universel, le groupoïde $\vec{G} \times M \rightrightarrows M$ est isomorphe à $\Pi_{1}\left(\mathcal{F}_{\phi}\right)$. Dans ce cas il existe un difféomorphismee local, $h: G \times M \longrightarrow H\left(\mathcal{F}_{\phi}\right)$, qui est un difféomorphisme si l'action de $G$ est libre [Plii]. La factorisation entre ces deux groupoïdes est donc déterminée par le diagramme suivant:

$$
\tilde{G} \times M \cong \Pi_{1}\left(\mathcal{F}_{\phi}\right) \frac{g}{\stackrel{f}{\downarrow} G \times M \underset{M}{\longrightarrow} \stackrel{h}{\longrightarrow}} H\left(\mathcal{F}_{\phi}\right)
$$

où $f$ est un revêtement et $h$ un difféomorphisme local.

Exemple. En particular l'action de $\mathcal{C}$ sur $\mathbb{C}^{*}$ :

$$
\begin{array}{ccc}
\mathbb{C} \times \mathcal{C}^{*} & \longrightarrow C^{*} \\
(z, \zeta) & \longrightarrow e^{z} \zeta,
\end{array}
$$

définit sur $\mathrm{C} \times \mathrm{C}^{*}$ une structure de groupoïde de Lie, qui est le groupoïde fondamental de la varieté $\mathrm{C}^{*}$.

Le rôle du groupoïde d'holonomie est joué par le groupoïde trivial $C^{*} \times C^{*} \rightrightarrows C^{*}$, et la factorisation est determinée par le morphisme $g$ :

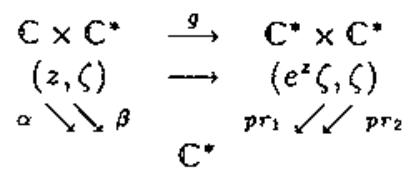


(b) Feuilletages induits sur des ouverts.

Soit un feuilletage ( $M, \mathcal{F}), W$ un ouvert de $M$ (pas nécessairement saturé) et $\mathcal{F}_{W}$ la restriction de $\mathcal{F}$ à $W$. L'inclusion $j: W \longrightarrow M$ induit l'homomorphisme injectif:

$$
\begin{aligned}
j^{*}: C\left(\mathcal{F}_{W}\right) & \longrightarrow C(\mathcal{F}) \\
\gamma & \longrightarrow j \circ \gamma
\end{aligned}
$$

Si deux chemins $\gamma_{1}$ et $\gamma_{2}$ son homotopes sur une feuille de $\mathcal{F}_{W}$, il en est de même sur la feuille de $\mathcal{F}$ qui les contient. La réciproque est vraie si $W$ est un ouvert saturé. L'homomorphisme $\Pi_{1}(j): \Pi_{1}\left(\mathcal{F}_{W}\right) \longrightarrow \Pi_{1}(\mathcal{F})$ est donc injectif si $W$ est un ouvert saturé. En général l'image de $\Pi_{1}(j)$ :

$$
\operatorname{Im} \Pi_{1}(j)=\left\{(\gamma] \in \Pi_{1}(\mathcal{F}) / \gamma \subset W\right\}
$$

est un sous-groupoïde ouvert de $\Pi_{1}(\mathcal{F})$, puisque $W$ est ouvert dans $M$.

Quant au groupoïde d'holonomie, deux chemins $\gamma_{1}$ et $\gamma_{2}$ ont le même germe d'holonomie dans $W$ si et seulement si ils ont le même germe dans $M$. L'homomorphisme $H(j): H\left(\mathcal{F}_{W}\right) \longrightarrow H(\mathcal{F})$ est, donc, toujours injectif et l'image de $H(j)$ est un sous groupoïde ouvert de $H(\mathcal{F})$ isomorphe à $H\left(\mathcal{F}_{W}\right)$.

Dans l'exemple ci-dessus l'inclusion $j: C^{*} \longrightarrow C$ induit l'homomorphisme $\Pi_{1}(j):$

$$
\begin{aligned}
& \Pi_{1}(j): \Pi_{1}\left(\mathcal{C}^{*}\right) \cong \mathbb{C}^{*} \times C \longrightarrow \Pi_{1}(\mathcal{C}) \cong \mathcal{C} \times C \\
& (z, \zeta) \rightarrow \quad\left(e^{z} \zeta, \zeta\right) \\
& \alpha \downarrow \beta \quad p r_{1} \downarrow \downarrow p r_{2} \\
& \mathrm{C}^{*} \longrightarrow \mathrm{C}
\end{aligned}
$$

Le noyau de $\Pi_{1}(j)$ est le sous-groupoïde Iso $\Pi_{1}\left(C^{*}\right)$, donc $\operatorname{Im} \Pi_{1}(j) \cong$ $\frac{\Pi_{1}\left(C^{*}\right)}{I \text { so } \Pi_{1}\left(C^{*}\right)}$ qui est un sous-groupoïde ouvert dans $\Pi_{1}(C)$. 
(c) Feuilletages définis par des fibrations localement triviales.

Si le feuilletage $\mathcal{F}$ sur $M$ est une fibration localement triviale de fibre $F$, alors:

i) Le groupoïde $\Pi_{1}(\mathcal{F}) \rightrightarrows M$ est une fibration localement triviale de fibre $\tilde{F}$ (revêtement universel de $F$ ).

ii) Le groupoïde $H(\mathcal{F}) \rightrightarrows M$ est aussi une fibration localement triviale de fibre $F$.

(d) Recollement de feuilletages.

Soit $M$ une variété à bord et $\mathcal{F}$ un feuilletage sur $M$ pour lequel $\partial M$ est une sous-variété saturée. Il est immédiat de vérifier que si l'on note $\partial \mathcal{F}=\left.\mathcal{F}\right|_{a M}$, alors le groupoide $\Pi_{1}(\mathcal{F})$ (resp. $H(\mathcal{F})$ ) est une variété à bord et on a $\Pi_{1}(\partial \mathcal{F}) \cong$ $\partial \Pi_{1}(\mathcal{F})($ resp. $H(\partial \mathcal{F}) \cong \partial H(\mathcal{F}))$.

En plus on a la proposition suivante:

1.3.1. Proposition. Soient $M_{1}$ et $M_{2}$ deux variétés à bord avec des feuilletages $\mathcal{F}_{1}$ et $\mathcal{F}_{2}$ tangents au bord et soit $f: \partial M_{1} \rightarrow \partial M_{2}$ un difféomorphisme qui préserve les feuilletages. Soit $M \cong M_{1} \amalg_{f} M_{2}$ et $\mathcal{F}$ le feuilletage induit sur M. Alors:

i) il existe une application $\Pi_{1}(f): \partial \Pi_{1}\left(\mathcal{F}_{1}\right) \longrightarrow \partial \Pi_{1}\left(\mathcal{F}_{2}\right)$, induite par $f$, qui est un difféomorphisme;

ii) le groupoüde $\Pi_{1}(\mathcal{F})$ est isomorphe $\dot{a} \Pi_{1}\left(\mathcal{F}_{1}\right) \Pi_{\Pi_{1}(f)} \Pi_{1}\left(\mathcal{F}_{2}\right)$.

Démonstration. Puisque f est un difféomorphisme qui préserve les feuilletages, $\Pi_{1}(f)$ est bien défni et est aussi un difféomorphisme.

Considérons sur la somme disjointe $M_{1} \amalg M_{2}$ la relation d'équivalence $R$ suivante: $x R x^{\prime}$ si et seulement si $x^{\prime}=f(x)$. Le quotient pour celte relation est difféomorphe $\dot{a} M$. La relation $\tilde{R}$ induite sur $\Pi_{1}\left(\mathcal{F}_{1}\right) \amalg \Pi_{1}\left(\mathcal{F}_{2}\right)$ est: $\gamma \tilde{R} \gamma^{\prime}$ si et seulement si $\gamma^{\prime}=f_{0} \gamma=\Pi_{1}(f)(\gamma)$. Si l'on note $p$ la projection on a le diagramme commutatif suvant:

$$
\begin{array}{ccc}
M_{1} \amalg M_{2} & \stackrel{p}{\longrightarrow} M_{1} \amalg_{f} M_{2} \cong M \\
\dot{\alpha} \downarrow \dot{\beta} & & \alpha \downarrow \beta \\
\Pi_{1}\left(\mathcal{F}_{1}\right) \amalg \Pi_{1}\left(\mathcal{F}_{2}\right) \stackrel{\Pi_{1(p)}}{\longrightarrow} & \Pi_{1}(\mathcal{F})
\end{array}
$$

$L$ homomorphisme $\Pi_{1}(p)$ est injectif et en plus si $\gamma, \gamma^{\prime} \in \Pi_{1}\left(\mathcal{F}_{1}\right) \amalg \Pi_{1}\left(\mathcal{F}_{2}\right)$ sont tels que $\Pi_{1}(p)(\gamma)=\Pi_{1}(p)\left(\gamma^{\prime}\right)$ alors $p_{0} \gamma=p_{0} \gamma^{\prime}$, c'est-i்-dire $\gamma^{\prime}=$ $\Pi_{1}(f) \cdot(\gamma)$. Donc $\Pi_{1}(\mathcal{F}) \cong \Pi_{1}\left(\mathcal{F}_{1}\right) \amalg_{\Pi_{1}(f)} \Pi_{1}\left(\mathcal{F}_{2}\right)$.

Remarque. On verra sur un exemple au paragraphe 3 qu'on n'a pas ce résultat, en général, pour le groupoïde d'holonomie. 
1.4. Séparation de $\Pi_{1}(\mathcal{F})$ et $H(\mathcal{F})$.

En général ni le groupoïde fondamental ni le groupoïde d'holonomie d'un feuilletage $\mathcal{F}$ ne sont separés. Pour le premier on a une caractérisation en termes de cycles évanouissants. Pour le second on verra le rapport avec le pseudogroupe d'holonomie associé.

Une application continue $h: X \rightarrow M$ est intégrale relativement à $\mathcal{F}$ si, $X$ étant connexe, l'image de $h$ est contenne dans une feuille de $\mathcal{F}$.

1.4.1. Définition. Un lacet intégral $\gamma: \$^{1} \longrightarrow M$ est un cycle évanouissant de $\mathcal{F}$ s'il existe une homotopie $A: S^{1} \times[0,1] \longrightarrow M$ de lacets integraux telle que $A_{0}=\gamma$ et $A_{i}$ est homotope à zéro sur la feuille $\mathcal{F}_{0}$ qui le contient pour tout $t>0$.

C'est un cycle évanouissant trivial si $\gamma=A_{0}$ est homotope à zéro sur la feuille $\mathcal{F}_{0}$.

1.4.2. Proposition. Soit $(M, \mathcal{F})$ un feuilletage régulier, on a les deux conditions équivalentes:

i) $\Pi_{1}(\mathcal{F})$ est séparé;

ii) tout cycle évanouissant de $\mathcal{F}$ est trivial.

Démonstration. Le fait que i) implique ii) est immédiat car si $\gamma: \$^{1} \longrightarrow$ $M$ est un cycle évanouissant non trivial de point de base $u_{0}=\gamma(1)$, alors les classes d'homotopie correspondantes $[\gamma]$ et $\left[u_{0}\right]$ ne sont pas separées : si $U$ et $V$ sont des voisinages de $[\gamma]$ et $\left[\chi_{\theta}\right]$ il existe $\varepsilon>0$ tel que $\left[A_{t}\right] \in U \cap V$ pour tout $t<\varepsilon$.

Pour la réciproque, soient $\gamma$ et $k$ deux lacets intégraux tels que $[\gamma]$ et $[k]$ soient non séparées dans $\Pi_{1}(\mathcal{F})$. Puisque l'espace des unités $M$ est separé alors $\gamma$ et $k$ ont les mêmes extrémités et quitte à les remplacer par $k_{0}^{-1} \gamma$ et $k^{-1} \cdot k$ on peut supposer que $k$ est le lacet constant $\gamma(1)$. Soient alors $U$ et $V$ des voisinages contractiles de $[\gamma]$ et $[k]$ (tous les éléments de $V$ étant des lacets constants), alors $U \cap V \neq \phi$ et il existe une famille à un parametre $A: \$^{1} \times[0,1] \longrightarrow M$ de lacets intégraux telle que:

i) $\left[A_{t}\right] \in U$ pour tout $t \in[0,1]$,

ii) $A_{0}=\gamma$ et $\left[A_{1}\right] \in V$, c'est-à-dire $A_{1}$ est homotope à zéro sur la, feuille $F_{1}$ qui le contient.

On considère $K=\left\{t \in[0,1] / A_{i}\right.$ est homotope à zéro sur la feuille $\left.F_{t}\right\}$. Par construction $1 \in K$ et la composante connexe de 1 dans $K, K_{1}$, est un ouvert d'après la trivialité locale des feuilletages. Si en plus tout cycle évanouissant de $\mathcal{F}$ est trivial, alors $K_{1}$ est aussi fermée, donc égale à $[0,1]$. D'où le résultat.

Maintenant on va caractériser les cas pour lesquels $H(\mathcal{F})$ est separé à l'aide du pseudogroupe d'holonomie.

1.4.3. Définition. Soit $f$ un difféomorphisme local de $\mathbf{R}^{p}$. On dira que $f$ est de type analytique si $f$ est l'identité lorsqu'il l'est sur un ouvert de son domaine. 
On dira qu'un pseudogroupe de difféomorphismes locaux, $G$, est de type analytique si chaque élémet de $G$ est de type analytique.

Remarque. Si $G$ est un pseudogroupe de type analytique alors tout pseudogroupe localement isomorphe est aussi de type analytique.

1.4.4. Définition. Soit $(M, \mathcal{F})$ un feuilletage régulier. On dira que $\mathcal{F}$ est transversalement de type analytique si le pseudogroupe d'holonomie de $\mathcal{F}$ est de type analytique.

1.4.5. Proposition. Soit $(M, \mathcal{F})$ un feuilletage régulier. Alors $H(\mathcal{F})$ est separé si et seulement si $\mathcal{F}$ est transversalement de type analytique.

Démonstration. On suppose d'abord que $H(\mathcal{F})$ est separé, et soit $f$ un élément du pseudogroupe d'holonomie pour lequel il existe un ouvert $U$ qui vérifie $\left.f\right|_{U}=I d$. Soit $x$ un point dans la fermeture de $U$. Alors il existe un lacet $\gamma$, de point de base $x$, tangent à $\mathcal{F}$, pour lequel le germe d'holonomie est $f$. Maintenant dans tout voisinage de la classe de $\gamma$ il y a des classes de la. cets constants, donc la classe de $\gamma$ est la classe constante et le difféomorphisme $f$ est l'identité.

Réciproquement, sí $x$ et $x^{\prime}$ sont deux éléments dans $H(\mathcal{F})$ non separés on peut supposer qu'bls sont des classes de lacets et que $x^{\prime}$ est la classe constante. Si dans tout voisinage de $x$ il existe des classes de lacets constants, alors il existe un ouvert sur lequel le germe d'holonomie de $x$ est l'identité. D'aprés l'hypothèse l'holonomie de $x$ est l'identité, donc $x=x^{\prime}$.

\section{Groupoïde fondamental du feuilletage $\left(M^{3}, \mathcal{F}\right)$ avec des cycles evanouissants non triviaux}

Soit $M$ une variété compacte de dimension 3 et $\mathcal{F}$ un feuilletage de codimension 1 orienté. S'il existe des cycles évanouissants non triviaux pour $\mathcal{F}$, alors d'après le théorème de Novikov [No], il existe un nombre fini de composantes de Reeb compactes. En plus, pour chaque $F \in \mathcal{F}, \pi_{2}(F)=0$, puisque si $\mathcal{F}$ avait une feuille sphérique, par le théorème de stabilité globale de Reeb [Ree] on pourrait conclure que ou bien $M \cong S^{2} \times S^{3}$ ou bien $M \cong S^{2} \times[0,1]$, feuilletée par $\$^{2}$ et sans composantes de Reeb.

\subsection{Groupoïde fondamental d'une composante de Reeb compacte.}

Soit $(W, R)$ une composante de Reeb compacte, c'est-à-dire $W \cong \mathrm{S}^{1} \times D^{2}$. On sait déjà que $\Pi_{1}(R)$ n'est pas separé et on va le décrire à l'aide d'un recouvrement par deux sous-groupoïdes ouverts, dont́ l'un n'est pas separé.

On notera $A$ l'âme de la composante, c'est-à-dire $A \cong \$^{1} \times\{0\}$.

\section{Premier sous-groupoïde.}

Soit $W_{1}=$ int $(W)$ et $R_{1}=\left.R\right|_{W_{1}}$ qui est une fibration triviale par plans au-dessus $\mathbf{S}^{1}$, donc $\Pi_{1}\left(R_{1}\right) \rightrightarrows W_{1}$ est aussi une fibration triviale par plans. 
Puisque $\left(W_{1}, R_{1}\right)$ est un ouvert saturé dans $(W, R)$ alors $\Pi_{I}\left(R_{1}\right)$ est un sousgroupoïde ouvert dans $\Pi_{1}(R)$.

Le sous-groupoïde $\Pi_{1}\left(R_{1}\right) \cong \mathrm{C} \times W_{1}$ est le premier sous-groupoïde du recouvrement.

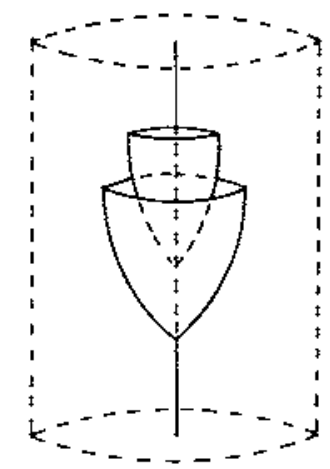

Sous-groupoïde intermédiaire.

On va considérer maintenant l'ouvert $I=W_{1} \mid A$ qui correspondra à l'intersection. Le feuilletage $R$ restreint à $I, R_{I}$, est une fibration triviale de fibre $C^{*}$ au-dessus de $S^{1}$, donc $\Pi_{1}\left(R_{1}\right) \Rightarrow I$ est une fibration triviale de fibre $C$.

I n'est pas saturé dans $W_{1}$ et d'une façon tout à fait analogue à celle de l'exemple de la première partie, on conclut que le noyan de l'homomorphisme $\Pi_{1}(j)$ induit par l'inclusion:

$$
\begin{aligned}
\Pi_{1}(j): \mathbb{C}^{*} \times \$^{1} \times C & \longrightarrow C \times \$^{1} \times \mathbb{C} \\
(z, \theta, \zeta) & \longrightarrow\left(e^{2} \zeta, \theta, \zeta\right)
\end{aligned}
$$

est isomorphe à Iso $\Pi_{1}\left(R_{I}\right)$. Donc $\operatorname{Im} \Pi_{1}(j) \cong \frac{\Pi_{1}\left(R_{I}\right)}{1 \text { so } \Pi_{1}\left(R_{I}\right)}$ est un sous-groupoïde ouvert dans $\Pi_{1}\left(R_{l}\right)$, donc il est aussi ouvert dans $\Pi_{1}\left(R_{I}\right)$ et separé.

Second sous-groupoïde.

Considerons $W_{2}=W \mid A$ et $R_{2}=\left.R\right|_{W_{2}}$ 
2.1.1. Proposition. Le groupoide $\Pi_{1}\left(R_{2}\right) \rightrightarrows W_{2}$ est une fibré localement trivial de fibre $\mathbb{C}$.

Démonstration. Sur $\left(W_{2}, R_{2}\right)$ il n'existe plus de cycle évanouissant non truvial, et $\pi_{2}(F)=0 \forall F \in R_{2}$. Si on considére le revêtement universel $p: \grave{W}_{2} \longrightarrow W_{2}$, le feuilletage induit sur $\tilde{W}_{2}$ est un feuilletage par plans, défni par l'action libre de $\mathrm{C} / \mathrm{Pal} /$. Le groupoide fondamental de $p^{*}\left(R_{2}\right)$ est trivial, isomorphe $a \dot{C} \times \tilde{W}_{2}$. Le groupe $A u t(p)$ agit sur $\mathcal{C} \times \tilde{W}_{2}$ comme groupe d'automorphismes de groupoide et de fibré, et préserve la fibre. Le quotient par cette action est $\Pi_{1}\left(W_{2}\right)$. Donc quitte à quotienter par $A$ ut $(p)$ on obtient le résultat cherché.

$\left(W_{2}, R_{2}\right)$ est un ouvert non sature dans $(W, R)$ et l'homomorphisme $\Pi_{1}(i): \Pi_{1}\left(R_{2}\right) \longrightarrow \Pi_{1}(R)$ n'est pas injectif. $D$ 'autre part le groupoïde Im $\Pi_{1}(i)$ est un sous-groupoide ouvert dans $\Pi_{1}(R)$ qui sera le second sons-groupoide du recouvrement. On va décrire Ker $\Pi_{1}(i)$ : l'ouvert $I$ est saturé dans $W_{2}$ et $W_{2} \mid I$, le bord de la composante de Reeb, est aussi saturé dans $W$. Donc, étant données les applications

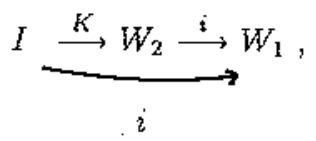

Ker $\Pi_{1}(i)$ est isomorphe à Ker $\Pi_{1}(l)$, donc isomorphe da Iso $\Pi_{1}\left(R_{I}\right) \subset I$ Iso $\Pi_{1}\left(R_{2}\right)$ On a $I m \Pi_{1}(i) \cong \frac{\Pi_{1}\left(R_{2}\right)}{\operatorname{Iso} \Pi_{1}\left(R_{1}\right)}$.

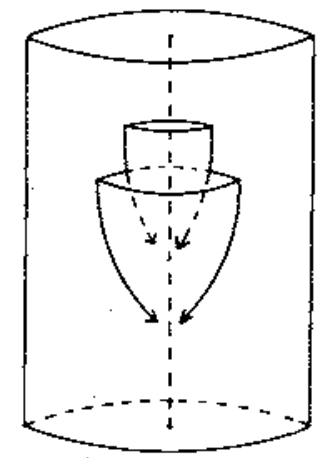

2.1.2. Théorème. Soit $(W, R)$ une composante de Reeb compacte de dimension 3. Soitnt $W_{1} \cong$ int $(W), W_{2} \cong W \mid A$ et $I \cong W_{1} \cap W_{2}$, et $R_{1}, R_{2}, R_{I}$ les feuilletages correspondants induits. Alors:

i) $\Pi_{1}\left(R_{1}\right) \rightrightarrows W_{1}$ est un fibré trivial de fibre $\mathrm{C}$;

ii) $\Pi_{1}\left(R_{2}\right) \rightrightarrows W_{2}$ est un fibré localement trivial de fibre $\mathrm{C}$;

iii) la famille $\left\{\Pi_{1}\left(R_{1}\right), \frac{\Pi_{1}\left(R_{2}\right)}{\text { Iso } \Pi_{1}\left(R_{T}\right)}\right\}$, est un recouvrement de $\Pi_{1}(R)$ par des sous-groupoïdes ouverts.

Démonstration. Il ne reste à vérifier que la famille de (iii) est un recouvrement de $\Pi_{1}(R)$. Soit $[\gamma] \in \Pi_{1}(R)$ et $\gamma$ un représentant de la classe. Si 
$\gamma \cap W_{1} \neq \phi$, puisque $W_{1}$ est saturé, $\gamma \subset W_{1}$ et la classe d'homotopie par rapport a $R$ est la même que par rapport a $R_{I}$, donc $[\gamma] \in \Pi_{1}\left(R_{1}\right)$. Si par contre $\gamma \cap W_{1}=\phi$, alors $\gamma \subset W\left|W_{1} \cong W_{2}\right| I$ qui est encore saturé et $\gamma \cap I=\phi$, donc $[\gamma] \in \frac{\Pi_{1}\left(R_{2}\right)}{\text { Iso } \pi_{1}\left(R_{Y}\right)}$.

Remarque. Le sous-groupoide $\frac{\Pi_{2}\left(R_{2}\right)}{\text { Iso } \Pi_{1}\left(R_{I}\right)}$ n'est pas separé car c'est le quo-' tient de $\Pi_{1}\left(R_{2}\right)$, qui est separé, par Iso $\Pi_{1}\left(R_{1}\right)$, qui est ouvert dans Iso $\Pi_{1}\left(R_{2}\right)$; on identifie les lacets qui, avec le même point de base, sont contenus dans linterieur de $\Pi_{3}\left(R_{2}\right)$. Les éléments son separés sont sur le bord de $\Pi_{1}\left(R_{2}\right)$.

En plus si $x, x^{\prime} \in \frac{\Pi_{1}\left(R_{2}\right)}{1 \text { so } \Pi_{1}\left(R_{I}\right)}$ sont deux éléments non separés, quitte à les remplacer par $x \cdot x^{1^{-1}}$ et $x^{1} \cdot x^{1^{-1}}$ on peut supposer que $x^{\prime}$ est le lacet constant $k=[\alpha(x)]$. Dans tout voisinage de $x$ il existe des classes de lacets constants. Donc $x$ est la classe d'homotopie du cycle évanouissant non trivial.

\subsection{Cas général.}

Il existe une façon générique de construire le groupö̈de fondamental d'un feuilletage avec des feuilles compactes en coupant à travers ces feuilles et en recollant les groupoïdes correspondants aux différents morceaux (prop. 1.3.1.). On peut aussi décrire le groupoïde $\Pi_{1}(\mathcal{F})$, si sur $\mathcal{F}$ il y a un nombre fini de composantes de Reeb $\left(W^{i}, R^{i}\right) i: 1, \ldots, n$, en généralisant le cas précédent,

On notera $A^{i}$ l'âme de chaque composante, $W_{1}^{i}=$ int $\left(W^{i}\right)$, $W_{2}^{i}=W^{i}\left|A^{i}, I^{i}=W_{1}^{i}\right| A^{i}, M_{2}=M \mid A_{1} U \ldots U A_{n}$, et $R_{1}^{i}, R_{I}^{i}, \mathcal{F}_{2}$ les feuilletages induits respectifs. Puisque $I^{i} \cap I^{j}=\phi$ si $i \neq j$ alors les sous-groupoïdes Iso $\Pi_{1}\left(R_{I}^{i}\right)$ sont deux à deux disjoints, et l'union disjonte $\bigsqcup_{i=1}^{n}$ Iso $\Pi_{1}\left(R_{I}^{i}\right)$, qu'on notera Iso $\Pi_{1}(I)$, est encore un sous-groupoïde de $I_{s o} \Pi_{1}\left(\mathcal{F}_{2}\right)$. On peut donc, considérer le quotient $\frac{\Pi_{1}\left(\mathcal{F}_{2}\right)}{\text { Iso } \Pi_{2}(I)}$.

La démonstration du théorème suivant est analogue à celle de 2.1.2.:

\subsubsection{Théorème. Dans ces conditions:}

i) pour chaque $i: 1, \ldots, u, \Pi_{1}\left(R_{1}^{i}\right) \rightrightarrows W_{1}^{i}$ est un fibré trivial de fibre $\mathrm{C}$;

ii) $\Pi_{1}\left(\mathcal{F}_{2}\right) \rightrightarrows M_{2}$ est un fibré localement trivial de fibre $C_{\text {; }}$

iii) la famille $\left\{\Pi_{1}\left(R_{1}^{1}\right), \ldots, \Pi_{1}\left(R_{1}^{n}\right), \frac{\Pi_{1}\left(\mathcal{F}^{2}\right)}{1 \text { so } \Pi_{1}(I)}\right\}$ est un recouvrement de $\Pi_{1}(\mathcal{F})$ par des sous-groupoïdes ouverts.

Remarque. Le groupoïde $\frac{\Pi_{2}\left(\mathcal{F}_{2}\right)}{\operatorname{Iso} \Pi_{3}(l)}$ 'est pas separé: on identifie tous les lacets qui, avec le même point de base sont dans l'interierur de $\Pi_{1}\left(W_{2}^{i}\right), i$ : $I, \ldots, n$. Les éléments non separés sont sur le bord de $\Pi_{1}\left(W_{2}^{i}\right)$. Comme dans la remarque du théorème précédent les éléments non separés son des cycles evanouissants de $\left(W^{i}, R^{i}\right)$. 


\section{Groupoïde d'holonomie du feuilletage de Reeb}

L'étude du groupoïde d'holonomie associé à un feuilletage régulier est différente de celle du groupoïde d'homotopie. En général on ne peut pas reconstruire le groupoïde global en recollant les groupoides de chaque morceau. Dans le premier paragraphe on va décrire le groupoïde d'holonomie de la composante de Reeb compacte, qui est separé. Il y a deux façons usuelles de recoller les bords de deux composantes: on obtient alors $\left(S^{2} \times S^{1}, \tilde{R}\right)$ on $\left(S^{3}, R^{\prime}\right)$. On verra que dans le premier cas le groupoide est le recollement des groupoïdes des morceaux, mais ce n'est plus vrai dans le second cas, pour lequel on obtient un groupoide d'holonomie non separé.

3.1. Groupoïde d'holonomie d'une composante de Reeb compacte.

Soit $(W, R)$ une composante de Reeb compacte, où $W \cong S^{1} \times D^{2}$. Soit $G=\left\{[x] \in\right.$ Iso $\Pi_{1}(R) /[x]$ n'est pas separé de $\left.\{\alpha(x)]\right\}$. Cést un sous groupoïde de Iso $\Pi_{1}(R)$ et le quotient $\frac{\Pi_{1}(R)}{G}$ est évidemment separé. En plus on sait par la proposition 1.4.5., puisque $R$ est transversalmente de type analytique, que $H(R)$ est aussi separé. Si $g: \Pi_{1}(R) \longrightarrow H(R)$ est la factorisation usuelle on a la proposition suivante:

3.1.1. Proposition. Dans les conditions antérieures il existe un difféomorphisme $\tilde{g}$ qui rend commutatif le diagramme suivant:

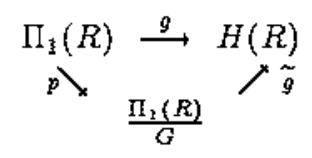

Démonstration. Il faut remarquer tout d'abord que dans ce cas $G$ est isomorphe à l'ensemble des cycles évanouissants non triviaux dans $(W, R)$. La classe d'homotopie est la même que la classe d'holonomie pour tout lacet que ne soit pas un cycle évanouissani non trivial, donc g est évidemment invariant par $G$. En plus, puisque g est un difféomorphisme local surjectif, il ne reste $\dot{a}$ verifier que $\tilde{g}$ est injectif. Mais ceci découle du fait que tout lacet avec holonomie triviale qui ne soit pas homotope à zéro sur la fenille qui le contient est un cycle évanouissant non trivial.

\subsection{Feuilletage de Reeb sur $S^{2} \times S^{2}$.}

Le difféomorphisme identité $f$ entre les bords de deux composantes de Reeb compactes induit, de façon naturelle, le difféomorphisme identité $f_{*}$ entre les chemins tangents à la feuille compacte:

$$
\begin{aligned}
f_{*}: C\left(\mathrm{~T}^{2}\right) & \longrightarrow C\left(\mathrm{~T}^{2}\right) \\
\gamma & \longrightarrow f_{0 \gamma}
\end{aligned}
$$

Sur la feuille compacte l'holonomie à gauche d'un chemin est la même qu'à droite (le groupoïde d'holonomie est donc separé), alors $f_{*}$ préserve les classes 
d'holonomie et induit un difféomorphisme $H(f)$ entre les bords des groupoïdes d'holonomie correspondants aux deux composantes. La démonstration de la proposition suivante est analogue à celle de 2.2.1.:

3.2.1. Proposition. Soient $\left(\$^{1} \times D^{2}, R_{1}\right)$ et $\left(\$^{1} \times D^{2}, R_{2}\right)$ deux composantes de Reeb compactes et $f$ le diffémorphisme identité entre les bords. Soit $\left(S^{1} \times D^{2}, \tilde{R}\right)$ le feuilletage résultant. Alors:

i) $f$ induit un difféomorphisme $H(f): H\left(\mathrm{~T}^{2}\right) \longrightarrow H\left(\mathrm{~T}^{2}\right)$;

ii) le groupoìde $H(\tilde{R})$ est isomorphe de $H\left(R_{3}\right) \amalg_{H(f)} H\left(R_{2}\right)$.

\subsection{Feuilletage de Reeb sur $\mathbb{S}^{3}$.}

Etant données deux composantes de Reeb compactes, si on identifie sur le bord les parallèles de l'une d'elles avec les méridiens de l'autre, on obtient le feuilietage de Reeb sur $\$^{3}:\left(\$^{3}, R^{\prime}\right)$. Le difféomorphisme $f_{*}$, induit sur les chemins tangents au bord, ne préserve pas les classes d'holonomie parce que sur la feuille corapacte l'holonomie à gauche n'est pas la même qu'à droite. On ne peut pas construire le groupoide $H\left(R^{\prime}\right)$ en recollant les groupoides de chaque composante.

D'autre part, la représentation d'holonomie de la feuille compacte est injective, donc $\operatorname{Im} H \cong \Pi_{1}\left(T^{2}\right)$, et on a le résultat suivant:

3.3.1. Proposition. Le groupoïde d'holonomie du feuilletage de Reeb sur $\$^{3}$ est isomorphe au groupoide d'homotopie.

Démonstration. Puisque pour toute feuille de $R^{\prime}$ la répresentation d'holo. nomie est injective, son image est difféomorphe au groupe fondamental de la feuille, et donc lo factorisation $g: \Pi_{1}\left(R^{\prime}\right) \longrightarrow H\left(R^{\prime}\right)$ est injective. Comme $g$ est toujours un difféomorphisme local surjectif, on déduit le résultat.

\section{References}

[He-Hi] G. HECTOR, U. HIRSCH, Introduction to the geometry of Foliations, Part A, B, Aspects of Mathematics.

[No] S. P. Novikov, Topology of Foliations, Trudy Mish. Math. Obsch. 14 (1965), 248-278. Trans. Moscow. Math. Soc. 268-304.

[Pal] C. F. B. PALMEIRA, Open manifolds foliated by planes, Annals of Math. 107 (1978), 109-131.

[Phi] J. PHILIPS, The holonomie imperative and the homotopy groupoid, Rocky Mountains J. of M. 17 (1987), 151-165.

[Ree] G. REEB, Sur certains propietés topologiques de variétés feuilletées, Act. Sc. Ind. 1183 (1952), 91-154, Herman Paris. 
[Win] H. E. WiNKELNKEMPER, The graph of a foliation, Ann. Glob. Analysis and Geometry 1, no. 3 (1983), 51-75.

Universidad del Pais Vasco

Facultad de Ciencias E.E. y E.E.

Avenida del Ejército, 87

48015 Bilbao, SPAIN

Rebut el 28 d'Abril de 1989 